\title{
High-pressure low-symmetry phases of cesium halides
}

\author{
Marco Buongiorno Nardelli \\ Laboratorio TASC dell'Istituto Nazionale di Fisica della Materia and BULL-Unix Competence Center, \\ AREA di Ricerca di Trieste, Padriciano 99, I-34012 Trieste, Italy \\ Stefano Baroni \\ Scuola Internazionale Superiore di Studi Avanzati (SISSA) Via Beirut 2/4, I-34014 Trieste, Italy \\ Paolo Giannozzi \\ Scuola Normale Superiore (SNS) Piazza dei Cavalieri 7, I-56126 Pisa, Italy
}

(Received 5 August 1994)

\begin{abstract}
The relative stability of different high-pressure phases of various cesium halides is studied from first principles and analyzed using the Landau theory of phase transitions. We present results for $\mathrm{CsI}, \mathrm{CsBr}$, and $\mathrm{CsCl}$ up to pressures of $\approx 100 \mathrm{GPa}$. A cubic-to-orthorhombic transition, driven by the softening of an acoustic phonon at the $M$ point of the Brillouin zone, is competing with the cubic-to-tetragonal martensitic transition typical of these compounds. The phonon softening takes place only in CsI and $\mathrm{CsBr}$ at a residual volume of $V / V_{0}=0.64,0.52$, respectively. A cubicto-tetragonal instability is found instead to occur at $V / V_{0} \approx 0.54$ for all the compounds considered here. The orthorhombic phase is stable only in CsI, whereas it is taken over by the tetragonal one in the case of $\mathrm{CsBr}$. Our analysis reveals the essential role played by the phonon-strain coupling in stabilizing the orthorhombic phase and in making the corresponding transition first order.
\end{abstract}

\section{INTRODUCTION}

Since the pioneering work of Madelung and Ewald, ${ }^{1}$ a huge amount of experimental and theoretical work has been devoted to alkali halides which are the simplest and most representative ionic solids. The interest in the high-pressure properties of these materials has been recently renewed by the introduction of the diamond anvil cell technology ${ }^{2}$ which has opened the way to exploring previously unaccessible portions of their phase diagram. CsI is the softest among alkali halides and it has the smallest optical gap. For these reasons it is an ideal candidate to display a pressure-induced bandoverlap metallization which is actually found at a pressure $P \approx 110 \mathrm{GPa}^{3}$ Looking for this effect, new and unexpected crystal phases have been found to be stable in the pressure range of a few tens GPa.

The first systematic studies of the high-pressure phases of cesium halides date back to 1984. From energydispersive x-ray diffraction experiments Huang et al. ${ }^{4}$ and Knittle et $a .^{5}$ were able to observe that CsI and $\mathrm{CsBr}$ (whose low-pressure stable phase is the cubic B2), undergo a transition which lowers their cubic symmetry when an applied pressure reduces their residual volume to $v=V / V_{0} \approx 0.54, V_{0}$ being the equilibrium volume. The observed transition pressures are of $\approx 39$ and $53 \mathrm{GPa}$ for $\mathrm{CsI}$ and $\mathrm{CsBr}$, respectively. ${ }^{5} \mathrm{~A}$ similar instability has been also reported in $\mathrm{CsCl}$, at about $65 \mathrm{GPa} .{ }^{4}$ This symmetry lowering manifests itself through the splitting of the (110) x-ray diffraction line into two peaks which are interpreted as corresponding to the (101) and (110) inequivalent reciprocal-lattice vectors of a tetragonal struc- ture. These authors did not observe any detectable discontinuity in any physical observable, and they conjectured therefore that the phase transition was of second order. However, general group-theoretical considerations indicate that a transition from a cubic to a tetragonal phase in which the only order parameter is the unit-cell shape, i.e., strain, cannot be of second order. ${ }^{6}$ We conclude therefore that a weak first-order character of the transition must have escaped the analysis of the experimental data. Claims that this tetragonal phase would undergo a further transition to an orthorhombic structure ${ }^{7}$ (space group $D_{2 h}^{1}$ ) were not confirmed by subsequent experimental and theoretical work. ${ }^{8-10}$

The first-order character of the cubic-to-tetragonal transition in cesium halides was confirmed by the very first theoretical investigations performed by Vohra et al. ${ }^{8}$ within a Born-Meyer semiempirical approach. According to these authors, the instability of the $\mathrm{CsCl}$ crystal structure is related to the softening of the shear constant, $c_{s}=\frac{1}{2}\left(c_{11}-c_{12}\right)$, due to the competition between the long-range Coulomb attraction and the short-range repulsive energy which becomes more and more important as the pressure increases. They also found that the tetragonal structure is stable against further orthorhombic distortions of symmetry corresponding to the space group $D_{2 h}^{1}$; i.e., for any given value of $a$ and $c / a$, the minimum of the lattice energy was always found at $b / a=1$ in the whole pressure range explored. Some investigations on CsI have also been carried out from first principles. The work of Christensen and Satpathy, ${ }^{9}$ performed within the linear muffin-tin orbital approximation, and that of Baroni and Giannozzi, ${ }^{10}$ in which $a b$ initio pseu- 
dopotentials were used, confirmed the overall picture proposed by Vohra et al. . $^{\text {. }}$

In 1989, new and unexpected features emerged from the x-ray diffraction experiments performed by Mao et $a l .{ }^{11}$ on CsI. Using a sophisticated experimental setup based on a synchrotron radiation source, they were able to identify a distortion of CsI from the cubic B2 to the hexagonal closed-packed structure passing through an intermediate orthorhombic phase whose assigned symmetry $\left(C_{2 v}^{1}\right)$, however, is different from that guessed in Ref. 7. It was observed that the (110) peak splits into three components, whereas two additional lines appear on the low- and high-energy sides of the triplet, corresponding to two diffraction peaks which are forbidden in the B2 structure. This diffraction pattern can be explained by assuming a $2 \times 1$ reconstruction of the cubic cell characterized by the gliding of one (110) plane, as depicted in Fig. 1. The onset of the phase transition, characterized by the broadening of the diffraction lines of the cubic structure, is observed at pressures as low as $15 \mathrm{GPa}$. The observed splittings and peak intensities evolve continuously from 15 to $100 \mathrm{GPa}$, showing a single orthorhombic phase with variable parameters. However, below $45 \mathrm{GPa}$ the splittings are not large enough to allow an unequivocal identification of the orthorhombic structure. The transition from the cubic to the orthorhombic phase was observed to be continuous within the experimental resolution.

In a recent paper ${ }^{12}$ we have shown that the distortion leading to the orthorhombic structure observed by Mao et $a .^{11}$ is driven by the softening of an $M_{5}^{-}$phonon at the $M$ point of the Brillouin zone (BZ). This softening is related to the ferroelastic instability responsible for the previously assumed cubic-to-tetragonal transition. The shear constant is in fact proportional to the square of the sound velocity along the (110) direction for vibrations polarized along (110). This observation suggests that a large reduction of the magnitude of the shear constant could favor the softening of a transverse phonon along the (110) direction. In fact, the gliding of the (110) plane depicted in Fig. 1 represents a lattice distortion which is very similar (but not strictly equal) to one of the two de-

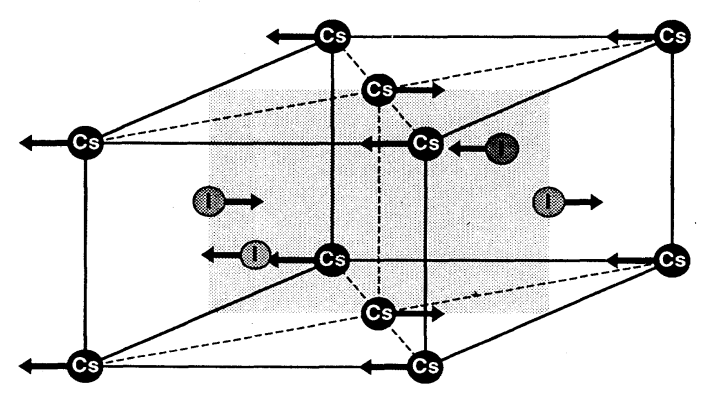

FIG. 1. Sketch of the CsI cell with the atomic displacements corresponding to an $M_{5}^{-}$acoustic phonon. The magnitude of cesium displacements with respect to iodine displacements is exaggerated for clarity. The solid lines indicate the cell of the distorted (orthorhombic) structure, while the cell of the undistorted (cubic) structure is indicated by a dashed line. The shaded area correspond to the (110) plane. generate ionic displacement patterns of the $M_{5}^{-}$acoustic phonon mode. We are thus led to identify the amplitude of this mode as the order parameter of the transition, in Landau's sense.

In this paper we present a complete account of our previous work on the high-pressure phases of CsI, ${ }^{12}$ and its extension to other cesium halides, $\mathrm{CsBr}$ and $\mathrm{CsCl}$. Our approach is based on a combined use of the Landau theory ${ }^{13}$ of phase transitions to classify the possible lowsymmetry phases and to determine the form of the interactions which can lead to them, and on first-principles calculations of the relevant interaction constants and energy differences as functions of the applied pressure. Our calculations are based on density-functional theory to determine crystal energies and their first derivatives with respect to atomic displacements (forces) and macroscopic strain (stress), and on density-functional perturbation theory to determine the second derivatives (essentially, the phonon frequencies). In Sec. II we present our theoretical framework and computational details. Section III contains our results. Section IV contains the conclusions.

\section{THEORY}

\section{A. Landau theory of phase transitions}

In the Landau theory of phase transitions, the relevant thermodynamical potential, $\mathcal{F}$, describing the relative stability of two phases is expressed as a power series of the so-called order parameter, $u$, whose value is different from zero in the low-symmetry (high-pressure, in the present case) phase, while $u$ vanishes in the highsymmetry (low-pressure) phase:

$$
\mathcal{F}(u)=\mathcal{F}_{0}+A_{2} u^{2}+A_{3} u^{3}+A_{4} u^{4}+\cdots,
$$

where the linear term in $u$ is missing to ensure the stability of the low-pressure phase. Let us suppose for the moment that the fourth-order coefficient, $A_{4}$, is positive so that it can ensure the global stability of the system, and let us neglect all higher-order terms. If $A_{2}>0$, the relative stability of the low- and highsymmetry phases is determined by the sign of the discriminant $\Delta_{6}=A_{3}^{2}-4 A_{2} A_{4}$. If $\Delta_{6}<0$ the highsymmetry $(u=0)$ phase is stable, while for $\Delta_{6}>0$ Eq. (1) has a minimum for $u \neq 0$ and the low-symmetry phase prevails. In this case, the transition from the highto the low-symmetry phase is discontinuous, and it is said to be first order. Continuous second-order transitions are possible if, because of symmetry, the third-order coefficient vanishes identically: $A_{3} \equiv 0$. In this case the highor low-symmetry phases are stable according to whether the second-order coefficient, $A_{2}$, is positive or negative. Suppose now that $A_{4}$ is negative in the region of the phase diagram where $A_{2}$ softens and that $A_{5} \equiv 0$. In this case, the global stability of the system must be ensured by the sixth- and higher-order terms and a first-order transition to the low-symmetry phase would then occur when $\Delta_{8} \equiv A_{4}^{2}-4 A_{2} A_{6}=0$.

For structural transitions where the order parameter is the amplitude of a lattice distortion, $A_{2}$ is essentially 
the square of the phonon frequency associated to that distortion. In the present case, the phonon which goes soft is the $M_{5}^{-}$mode at the $M$ point of the BZ. The small group of the $M$ point-whose coordinates are $\left(\frac{1}{2} \frac{1}{2} 0\right)$ - is $D_{4 h}$ and its star is made of three equivalent points. $M_{5}^{-}$ transforms according to a doubly degenerate irreducible representation of $D_{4 h}$, so that the order parameter associated with this mode is six dimensional: $\mathbf{u} \equiv\left\{u_{i}\right\}_{i=1,6}$. The $M_{5}^{-}$representation is odd with respect to inversion, so that no third-order (or, more generally, odd-order) terms must be present in Eq. (1) in order to ensure invariance with respect to the (cubic) symmetry group of the low-pressure phase. Following Landau's argument, we conclude that $A_{3} \equiv 0$ and $A_{5} \equiv 0$. Assuming that the sixth- and higher-order terms in Eq. (1) are positive in the neighborhood of the transition, this is then first or second order, according to whether the fourth-order coefficient is negative or positive, respectively.

\section{B. First-principles techniques}

In this paper we will ignore any entropy effects and restrict ourselves to zero temperature. In this condition, the implementation of the above phenomenological scheme requires the knowledge of the system energy as a function of the atomic displacements (phonon amplitudes) and unit-cell distortions (strain). Densityfunctional theory (DFT) is a very reliable and computationally viable tool for the study of the energetics of simple materials. We refer the reader to the many available review papers on this subject ${ }^{14}$ and we summarize here the main points. The local-density approximation (LDA) to DFT provides a practical, though approximate, way to map the ground-state properties of a system of interacting electrons onto those of a system of noninteracting electrons, subject to an effective external field which depends self-consistently on the ground-state electronic density. DFT-LDA allows one to calculate in a rather accurate and efficient way the energies of materials within the Born-Oppenheimer approximation, along with their first derivatives with respect to external parameters, such as, e.g., nuclear coordinates (i.e., atomic forces) (Ref. 15) and unit-cell shape and volume (i.e., stress). ${ }^{16}$ By linearizing the DFT-LDA equations with respect to the strength of an external perturbation, the second derivatives of the energy can be conveniently obtained by solving a suitable set of linear equations. ${ }^{17,18}$ This is the essence of the density-functional perturbation theory (DFPT) approach to lattice dynamics, which we will employ to determine the phonon spectra of the system considered, as functions of the crystal volume, i.e., of the applied pressure.

Our calculations are performed in the framework of the plane-wave pseudopotential method. ${ }^{14}$ For Cs and I we have used the same pseudopotentials as in Ref. 10. Pseudopotentials for $\mathrm{Br}$ and $\mathrm{Cl}$ have been generated using the method originally proposed by von Barth and Car. ${ }^{19}$ For Cs, electrons up to $4 d$ have been treated as frozen in the core. The inclusion of the Cs $5 s$ and $5 p$ states into the valence shell is necessary to obtain sensible results. In fact we found that a Cs pseudopotential which sustains only the $6 s$ electron as a valence electron yields a monotonic decrease of the crystal energy at decreasing volume, with no stability at all. An explicit account for the nonlinear core corrections, ${ }^{20}$ suggested by the large size of the Cs core, does not substantially improve the results. The inclusion of Cs $5 s$ and $5 p$ orbitals in the valence does not increase dramatically the numerical burden. In fact, the spatial extension of these orbitals is comparable to those of the anion $s$ and $p$ valence wave functions, so that the plane-wave basis set necessary to describe the latter is also adequate for the former.

Plane waves up to a kinetic energy cutoff of $25 \mathrm{Ry}$ were included in the basis set. The electron-gas exchangecorrelation energy and potential used to implement LDA are those determined by Ceperley and Alder ${ }^{21}$ as interpolated by Perdew and Zunger. ${ }^{22}$ Brillouin-zone integrations have been performed using sets of special points corresponding to the (666) Monkhorst-Pack mesh. ${ }^{23}$ This mesh-which corresponds to 10, 12, and 24 points in the cubic, tetragonal, and orthorhombic structures, respectively-has been explicitly checked for convergence for all the relevant structural properties of interest here.

\section{RESULTS}

\section{A. Zero-pressure properties}

The low-pressure structural properties of the materials investigated have been determined by fitting the calculated crystal energies to the Murnaghan equation of state. ${ }^{24}$ DFPT allows a straightforward determination of the electronic contribution to the static dielectric constants and Born effective charges. ${ }^{17}$ The theoretical predictions for these data are compared with experiments in Table I. The resulting accuracy on the predicted equilibrium lattice constants and bulk moduli is of the order of $3 \%$ and $10 \%$, respectively, i.e., in the typical range of DFT-LDA calculations. Dielectric constants are system-

TABLE I. Comparison between theoretical and experimental lattice constant, $a_{0}$, bulk modulus, $B_{0}$, and electronic static dielectric constant, $\epsilon_{\infty}$.

\begin{tabular}{lllll}
\hline \hline & & $a_{0}(\AA)$ & $B_{0}(\mathrm{GPa})$ & $\epsilon_{\infty}$ \\
\hline CsI & Theory & 4.44 & 15.4 & 3.68 \\
& Expt. & $4.56^{\mathrm{a}}$ & $13.5^{\mathrm{a}}$ & $3.02^{\mathrm{e}}$ \\
& & & & \\
$\mathrm{CsBr}$ & Theory & 4.17 & 20.7 & 3.36 \\
& Expt. & $4.28^{\mathrm{b}}$ & $17.9^{\mathrm{b}}$ & $2.83^{\mathrm{e}}$ \\
& & & & \\
$\mathrm{CsCl}$ & Theory & 3.99 & 24.3 & 3.16 \\
& Expt. & $4.12^{\mathrm{c}}$ & $22.9^{\mathrm{d}}$ & $2.67^{\mathrm{e}}$ \\
& & &
\end{tabular}

${ }^{\text {a Reference } 11 .}$

${ }^{\mathrm{b}}$ Reference 26.

${ }^{\mathrm{c}}$ Reference 27 .

${ }^{\mathrm{d}}$ Reference 28.

${ }^{\text {e }}$ Reference 29 . 


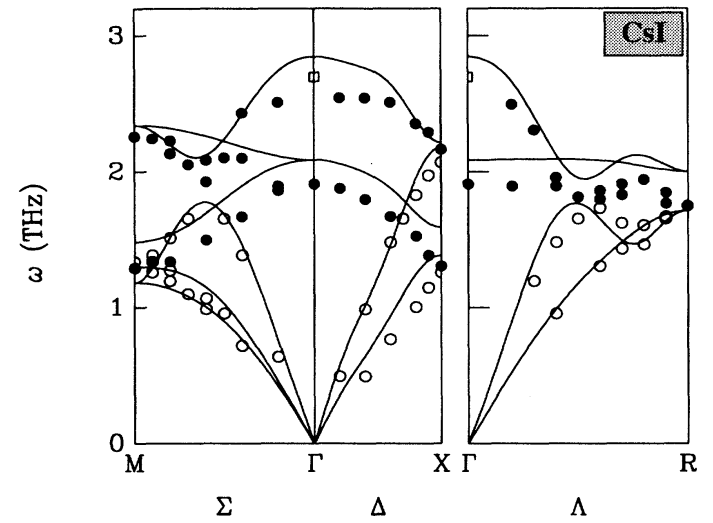

FIG. 2. Phonon dispersion relations along some symmetry directions of the Brillouin zone in CsI at equilibriumi volume. Experimental data indicated by open and black circles are from Ref. 30; an infrared measurement for $\omega_{\mathrm{LO}}(\Gamma)$ is indicated by an open square (Ref. 31).

atically overestimated by $\approx 20 \%$, a well-known drawback of the LDA. ${ }^{25}$ Using the theoretically determined equilibrium lattice parameters, the phonon dispersions of the three salts have been calculated by DFPT along the main symmetry directions. In Fig. 2 we display the calculated dispersion of CsI and compare them with neutrondiffraction data. Selected frequencies for CsI, CsBr, and $\mathrm{CsCl}$ are reported and compared with experiments in $\mathrm{Ta}-$ ble II. The agreement is quite satisfactory and gives us confidence in the predictive power of our calculations.

\section{B. Phonons at high pressure}

In order to substantiate the hypothesis of a mode softening occurring upon increasing pressure along the (110) acoustic branch, we have calculated the corresponding phonon dispersion for several values of the crystal residual volume, $v$. In Fig. 3 we display our results for CsI. The $M_{5}^{-}$acoustic phonon softens at $v^{*}=0.638$, corresponding to a pressure $P^{*} \approx 23 \mathrm{GPa}$, as a consequence of the incipient softening of the sound velocity of one of the transverse branches. The same behavior is observed

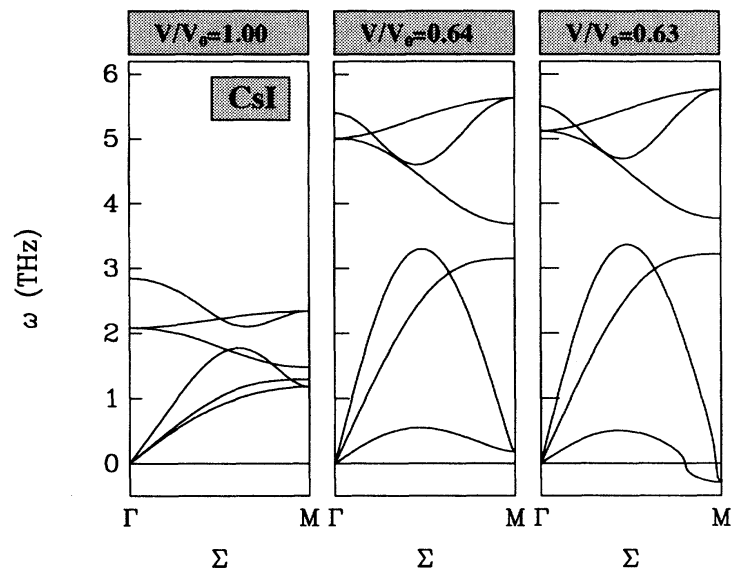

FIG. 3. Phonon dispersion relations along the $\Sigma$ (110) for CsI at equilibrium volume and just above and below the softening pressure of the $M_{5}^{-}$acoustic mode. "Negative" frequencies actually mean "imaginary" (i.e., negative squared frequencies).

in $\mathrm{CsBr}$ at $v^{*}=0.520$ while $\mathrm{CsCl}$ does not display any tendency to softening upon increasing pressure. The dependence of the $M_{5}^{-}$acoustic phonon frequency upon the residual volume is displayed in Fig. 4 for $\mathrm{CsI}, \mathrm{CsBr}$, and CsCl.

\section{High-pressure phases}

\section{Tetragonal phase}

As a first step we have reinvestigated the cubic-totetragonal transition described in the Introduction. In Fig. 5 we display the zero-temperature enthalpies vs the $c / a$ cell parameter calculated at constant pressure for $\mathrm{CsI}, \mathrm{CsBr}$, and $\mathrm{CsCl}$. In $\mathrm{CsI}$ and in $\mathrm{CsBr}$ the existence of a first-order transition is clearly visible with a second minimum occurring at $c / a>1$ above some critical pressure, $P_{\text {tet }}$, and separated from the high-symmetry phase by a well-defined enthalpy barrier. The transition pressures are $P_{\text {tet }}=44$ and $58 \mathrm{GPa}$ for CsI and $\mathrm{CsBr}$, respectively, with a very small volume discontinu-

TABLE II. Comparison between calculated and experimentally observed phonon frequencies of cesium halides at zero pressure, at the $\Gamma, M, X$, and $R$ points of the BZ. Units are THz. The uncertainty on the last figure in the experimental data is indicated in parentheses.

\begin{tabular}{|c|c|c|c|c|c|c|c|c|c|c|c|c|c|}
\hline & & $\omega_{\mathrm{To}}(\Gamma)$ & $\omega_{L O}(\Gamma)$ & $\omega_{\mathrm{TA}}(M)$ & $\omega_{\mathrm{LA}}(M)$ & $\omega_{\mathrm{TO}}(M)$ & $\omega_{\mathrm{LO}}(M)$ & $\omega_{\mathrm{TA}}(X)$ & $\omega_{\mathrm{LA}}(X)$ & $\omega_{\mathrm{TO}}(X)$ & $\omega_{\mathrm{LO}}(X)$ & $\omega_{\mathrm{LA}}(R)$ & $\omega_{\mathrm{LO}}(R)$ \\
\hline \multirow[t]{2}{*}{$\overline{\text { CsI }}$} & Theory & 2.09 & 2.85 & 1.17 & 1.30 & 1.48 & 2.35 & 1.38 & 1.59 & 2.18 & 2.21 & 1.71 & 2.00 \\
\hline & Expt. & $1.91(5)^{\mathrm{a}}$ & $2.74(1)^{b}$ & $1.29(6)^{\mathrm{a}}$ & $1.34(5)^{\mathrm{a}}$ & - & $2.26(5)^{\mathrm{a}}$ & $1.21(5)^{\mathrm{a}}$ & $1.30(6)^{\mathrm{a}}$ & $2.07(5)^{\mathrm{a}}$ & $2.16(6)^{\mathrm{a}}$ & $1.75(5)^{\mathrm{a}}$ & - \\
\hline \multirow[t]{2}{*}{$\mathrm{CsBr}$} & Theory & 2.44 & 3.53 & 1.27 & 1.48 & 1.98 & 2.79 & 1.47 & 2.15 & 2.42 & 3.00 & 1.89 & 2.63 \\
\hline & Expt. & $2.29(2)^{\mathrm{c}}$ & $3.39(2)^{b}$ & $1.22(3)^{\mathrm{c}}$ & $1.61(3)^{\mathrm{c}}$ & $1.77(5)^{\mathrm{c}}$ & $2.69(5)^{\mathrm{c}}$ & $1.32^{\mathrm{c}}$ & $1.91^{\mathrm{c}}$ & - & - & $1.89^{c}$ & $2.40^{\mathrm{c}}$ \\
\hline \multirow[t]{2}{*}{$\mathrm{CsCl}$} & Theory & 3.33 & 5.06 & 1.24 & 1.86 & 3.18 & 4.04 & 1.43 & 2.44 & 3.21 & 4.50 & 1.91 & 3.94 \\
\hline & Expt. & $3.17(2)^{\mathrm{d}}$ & - & $1.19(3)^{\mathrm{d}}$ & $1.90(2)$ & $2.77(2)^{\mathrm{d}}$ & $3.75(3)^{d}$ & $1.31^{\mathrm{d}}$ & $2.98^{\mathrm{d}}$ & - & - & $2.02^{d}$ & $3.85^{\mathrm{d}}$ \\
\hline
\end{tabular}

\footnotetext{
${ }^{a}$ Reference 30 .

${ }^{\mathrm{b}}$ Reference 31 .

${ }^{\mathrm{c}}$ Reference 32.

${ }^{\mathrm{d}}$ Reference 33 .
} 


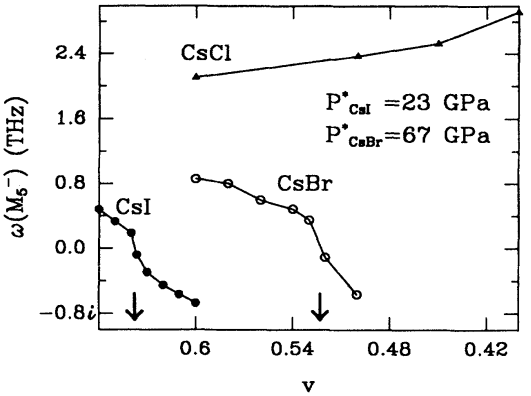

FIG. 4. Frequencies of the $M_{5}^{-}$acoustic phonon as functions of the molar volume for $\mathrm{CsI}, \mathrm{CsBr}$, and $\mathrm{CsCl}$. Arrows indicate the softening volume. $P^{*}$ is the softening pressure.

ity $(\approx 0.5 \%$ and $\approx 0.7 \%$, respectively $)$. In Fig. 6 we report the $c / a$ parameter vs $P / P_{\text {tet }}$ for CsI and CsBr. In $\mathrm{CsCl}$ the stability of the low-symmetry phase cannot be unambiguously established within our present accuracy. An instability towards the tetragonal phase is signaled by the flatness of the enthalpy as a function of $c / a$ for pressures $\approx 70 \mathrm{GPa}$. At higher pressures, however, this instability seems to weaken, and calculations for pressures as high as $100 \mathrm{GPa}$ do not give any evidence of a stable tetragonal phase.

\section{Orthorhombic phase}

As we have seen in Sec. II A, the vanishing of the $M_{5}^{-}$phonon frequency signals the onset of a phase transition whose order parameter is the phonon amplitude and which can be second-order because no third-order terms are present in the expansion of the crystal energy in powers of the order parameter. In order to investigate the character of the transition and determine the possible low-symmetry phases, it is necessary to find out the expression of the crystal energy in powers of $u$ to

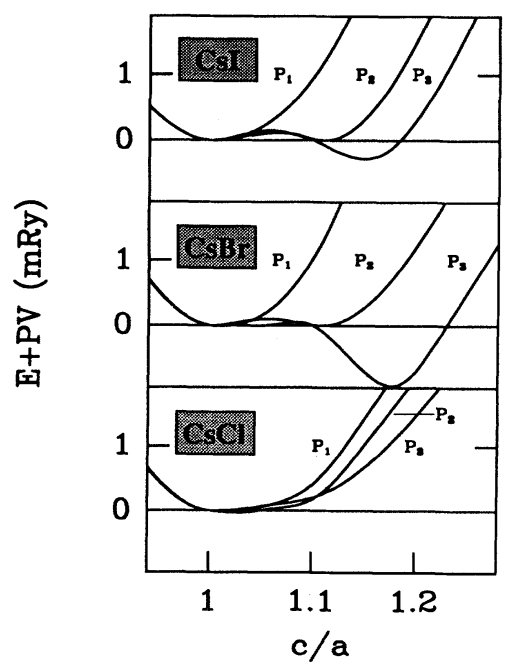

FIG. 5. Enthalpies vs $c / a$ for the three compounds corresponding to a pressure $P_{1}<P_{\text {tet }} ; P_{2} \approx P_{\text {tet }} ; P_{3}>P_{\text {tet }}$.

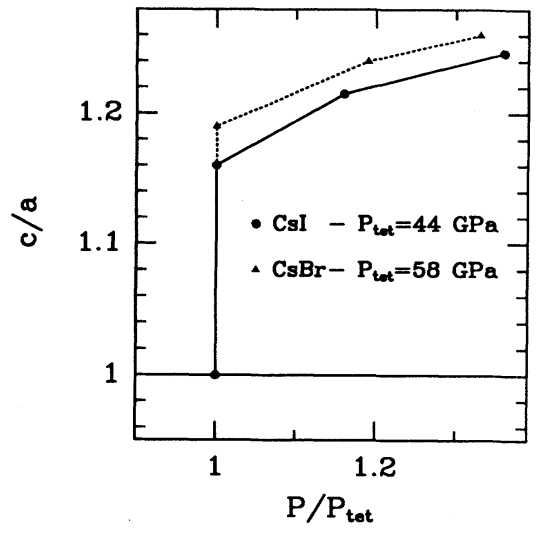

FIG. 6. $c / a$ parameter as a function of pressure for CsI and CsBr. $P_{\text {tet }}$ indicates the transition pressure to the tetragonal phase.

the lowest meaningful order, i.e., $n=4$. In Table III we display the wave vectors and the polarizations corresponding to one possible choice for the components of the six-dimensional order parameter. The corresponding fourth-order invariant polynomials can be obtained by using standard group-theoretical techniques. The $u$ 's realize an irreducible representation of the symmetry group of the high-symmetry phase (space group $O_{h}^{1}$ ). The number of fourth-order invariants is equal to the number of times the identical representation of $O_{h}$ is contained in the symmetric part of $\left[M_{5}^{-}\right]^{4}$. A simple exercise shows that this number is equal to four. A straightforward way to obtain them is to symmetrize all the possible translationally invariant fourth-order monomials with respect to $O_{h}$. In order to satisfy translational invariance, the starting monomials, $u_{i} u_{j} u_{k} u_{l}$ must satisfy the relation: $\mathbf{q}_{i}+\mathbf{q}_{j}+\mathbf{q}_{k}+\mathbf{q}_{l}=\mathbf{G}$, where $\mathbf{q}_{i}$ is the wave vector associated to the $i$ th component of the order parameter, and $\mathbf{G}$ is a reciprocal-lattice vector of the cubic phase. The four independent invariants are summarized in Table IV. The resulting expansion of the crystal energy reads

$$
\mathcal{F}(\mathbf{u})=\mathcal{F}_{0}+A_{2} \sum_{i=1,6} u_{i}^{2}+\sum_{i=1,4} A_{4}^{i} P_{4}^{i}(\mathbf{u})+\mathcal{O}\left(u^{6}\right)
$$

The possible low-symmetry stable phases compatible with this expression for the crystal energy have been worked out in Ref. 34. Following the notation of Ref. 34, these stable phases can be classified as in Table V, where each configuration corresponds to a particular direction in the six-dimensional order parameter space, i.e., to a particular combination of phonon displacements. In the

TABLE III. Phonon wave vectors, $\mathbf{q}$, and polarizations, e, of the six components of the order parameter, $\mathbf{u}$.

\begin{tabular}{ccccccc}
\hline \hline & $u_{1}$ & $u_{2}$ & $u_{3}$ & $u_{4}$ & $u_{5}$ & $u_{6}$ \\
\hline $\mathbf{q}$ & $\left(0 \frac{1}{2} \frac{1}{2}\right)$ & $\left(0 \frac{1}{2} \frac{1}{2}\right)$ & $\left(\frac{1}{2} 0 \frac{1}{2}\right)$ & $\left(\frac{1}{2} 0 \frac{1}{2}\right)$ & $\left(\frac{1}{2} \frac{1}{2} 0\right)$ & $\left(\frac{1}{2} \frac{1}{2} 0\right)$ \\
e & $(100)$ & $\left(0 \frac{1}{\sqrt{2}} \frac{1}{\sqrt{2}}\right)$ & $(010)$ & $\left(\frac{1}{\sqrt{2}} 0 \frac{1}{\sqrt{2}}\right)$ & $(001)$ & $\left(\frac{1}{\sqrt{2}} \frac{1}{\sqrt{2}} 0\right)$ \\
\hline \hline
\end{tabular}


TABLE IV. Fourth-order invariant polynomials which enter the free-energy expansion, Eq. (2).

\begin{tabular}{lc}
\hline \hline$P_{4}^{1}(\mathbf{u})$ & $u_{1}^{4}+u_{2}^{4}+u_{3}^{4}+u_{4}^{4}+u_{5}^{4}+u_{6}^{4}$ \\
$P_{4}^{2}(\mathbf{u})$ & $u_{1}^{2} u_{2}^{2}+u_{3}^{2} u_{4}^{2}+u_{5}^{2} u_{6}^{2}$ \\
$P_{4}^{3}(\mathbf{u})$ & $-u_{1} u_{2}\left(u_{3}^{2}+u_{4}^{2}-u_{5}^{2}-u_{6}^{2}\right)+$ \\
& $u_{3} u_{4}\left(u_{5}^{2}+u_{6}^{2}-u_{1}^{2}-u_{2}^{2}\right)+$ \\
$P_{4}^{4}(\mathbf{u})$ & $u_{5} u_{6}\left(u_{1}^{2}+u_{2}^{2}-u_{3}^{2}-u_{4}^{2}\right)$ \\
\hline \hline
\end{tabular}

specific case of CsI, the four fourth-order coefficients appearing in Eq. (2) have been fitted to the results of DFTLDA calculations performed for lattice distortions along some of the order-parameter directions listed in Table V. In fact, the coefficients of the crystal energy expansion are easily obtained through total energy and stress calculations. With the numerical values so obtained, we have verified that at the crystal volume where the $M_{5}^{-}$phonon frequency softens, the only minimum of the energy given by Eq. (2) occurs along the $P 11$ direction, whereas directional minima along all the other directions are actually saddle points. This observation seems to suggest that a second-order transition to a phase of tetrahedral symmetry $\left(T^{5}\right)$ would occur at the softening pressure of the $M_{5}^{-}$phonon. This finding is at variance with experimental data which indicate that the high-pressure phase has an orthorhombic symmetry, compatible with a distortion along the $P 1$ line.

The above considerations hold in the hypothesis that the strain state of the crystal is constant across the transition, except for the isotropic compression due to the application of a hydrostatic pressure. We have seen, however, that the softening of the $M_{5}^{-}$phonon mode is closely related to the softening of the shear constant of the crystal, so that a strong coupling between the soft mode and macroscopic strain (i.e., between zone-center and zoneborder acoustic phonons) is to be expected. Let us consider now the expression of the Landau crystal energy up to fourth order in the phonon amplitude, including the coupling with the anisotropic components of the strain tensor, $\epsilon$ :

$$
\mathcal{F}(\mathbf{u}, \epsilon)=\mathcal{F}(\mathbf{u}, \epsilon=0)+Q(\mathbf{u}, \epsilon),
$$

where $Q(\mathbf{u}, \epsilon)$ is a polynomial in $u$ and in the components of the strain tensor. Up to fourth order in $u Q(\mathbf{u}, \epsilon)$ contains only $\epsilon^{2}$ and $u^{2} \epsilon$ terms. In fact all odd terms in $u$ must vanish by symmetry, and $\epsilon$ is second order in

TABLE V. Classification of the possible stable phases along particular directions of the order-parameter space.

\begin{tabular}{lcc}
\hline \hline & Direction & Space group \\
\hline$P 1$ & $(1,0,0,0,0,0)$ & $D_{2 h}^{5}$ \\
$P 2$ & $(1,-1,0,0,0,0)$ & $D_{2 h}^{19}$ \\
$P 6$ & $(1,0,1,0,1,0)$ & $C_{3 v}^{5}$ \\
$P 7$ & $(0,-1,0,1,0,1)$ & $D_{3}^{7}$ \\
$P 9$ & $(1,-1,1,-1,0,0)$ & $D_{4 h}^{17}$ \\
$P 10$ & $(1,-1,0,0,1,-1)$ & $D_{4 h}^{17}$ \\
$P 11$ & $(1,-1,1,1,1,1)$ & $T^{5}$ \\
\hline \hline
\end{tabular}

$u$. To see this, we consider that, for a given value of $u$, the actual value of $\epsilon$ is that which minimizes the crystal energy given by Eq. (3). To leading order in $u$ one has

$$
Q(\mathbf{u}, \epsilon)=a \epsilon^{2}+b u^{2} \epsilon,
$$

the equilibrium condition $\partial Q / \partial \epsilon=0$ yields $\epsilon \propto u^{2}$. As a consequence other allowed terms such as $u^{2} \epsilon^{2}, \epsilon^{3}$, and $\epsilon^{4}$ are sixth or higher order in $u$.

To find the form of the $u^{2} \epsilon$ invariants we have first determined all the second-order monomials, $u_{i} u_{j}$, such that $\mathbf{k}_{i}+\mathbf{k}_{j}=\mathbf{G}$. There are nine such monomials which realize a reducible representation of the $O_{h}$ point group. The decomposition of this reducible representation into irreducible components is displayed in Table VI. The six independent components of the strain tensor split into three irreducible representation of the cubic group, as indicated in Table VII. The desired invariants are finally obtained by coupling each irreducible representation from the $u u$ set (Table VI), with an equal irreducible representation from the $\epsilon$ set (Table VII). There are four such invariants because the identical representation is contained four times in the direct product of the $u u$ and $\epsilon$ representations. The invariants are obtained as $Q_{4}^{i}(u, \epsilon)=\sum_{l} \epsilon_{l}^{i} \otimes u u_{l}^{i}$, where $i$ is the index of the irreducible representation and $l$ is the index of the basis function. These invariants are listed in Table VIII. The resulting expression for the crystal energy including the coupling with the strain reads

$$
\begin{aligned}
\mathcal{F}(\mathbf{u}, \epsilon)= & \mathcal{F}_{0}+A_{2} \sum_{i=1,6}{u_{i}}^{2}+\sum_{i, j=1}^{6} c_{i j} \epsilon_{i} \epsilon_{j} \\
& +\sum_{i=1,4} A_{4}^{i} P_{4}^{i}(\mathbf{u})+\sum_{i=1,4} B^{i} Q_{4}^{i}(\mathbf{u}, \epsilon)+\mathcal{O}\left(u^{6}\right),
\end{aligned}
$$

where $c_{i j}$ are the elastic constants, $\epsilon_{i}$ components of the strain tensor, and the $Q_{4}^{i}$ are the polynomials listed in Table VIII.

Numerical values of the $B$ coefficients have been determined along lines similar to those used to determine the $A_{4}$ 's. The $P 11$ minimum previously found to be stable ignoring the phonon-coupling strain is unaffected by such a coupling, while the minima along four out of the remaining six directions of Table $\mathrm{V}$ are slightly modified, still maintaining the saddle-point character they had in absence of such a coupling. The coupling to the strain changes instead the character of the directional minima

TABLE VI. Decomposition of the set of lattice-periodic quadratic monomials in the order parameter into irreducible representations of the cubic group.

\begin{tabular}{cc}
\hline \hline Label & Basis functions \\
\hline$\Gamma_{2}^{+}$ & $-u_{1} u_{2}+u_{3} u_{4}+u_{5} u_{6}$ \\
$\Gamma_{3}^{+}$ & $-2 u_{1} u_{2}-u_{3} u_{4}-u_{5} u_{6} ;$ \\
& $u_{3} u_{4}-u_{5} u_{6}$ \\
$\Gamma_{1}^{+}$ & $u_{1}^{2}+u_{2}^{2}+u_{3}^{2}+u_{4}^{2}+u_{5}^{2}+u_{6}^{2}$ \\
$\Gamma_{3}^{+}$ & $2\left(u_{1}^{2}+u_{2}^{2}\right)-u_{3}^{2}-u_{4}^{2}-u_{5}^{2}-u_{6}^{2} ;$ \\
& $u_{3}^{2}+u_{4}^{2}-u_{5}^{2}-u_{6}^{2}$ \\
$\Gamma_{5}^{+}$ & $u_{1}^{2}-u_{2}^{2} ; u_{3}^{2}-u_{4}^{2} ; u_{5}^{2}-u_{6}^{2}$ \\
\hline \hline
\end{tabular}


TABLE VII. Decomposition of the set of independent components of a symmetric rank-2 tensor into irreducible representations of the cubic group.

\begin{tabular}{cc}
\hline \hline Label & Basis functions \\
\hline$\Gamma_{1}^{+}$ & $\frac{1}{3}\left(\epsilon_{x x}+\epsilon_{y y}+\epsilon_{z z}\right)$ \\
$\Gamma_{3}^{+}$ & $\left.\epsilon_{x x}-\epsilon_{y y} ; 2 \epsilon_{z z}-\epsilon_{x x}-\epsilon_{x x}\right)$ \\
$\Gamma_{5}^{+}$ & $\epsilon_{x y} ; \epsilon_{y z} ; \epsilon_{z x}$ \\
\hline \hline
\end{tabular}

along $P 1$ and $P 2$. Let us consider the simpler case of the $P 1$ minimum. Projecting Eq. (5) along $P 1$ one obtains

$$
\begin{aligned}
\mathcal{F}^{P 1}\left(u, \epsilon_{s}, \epsilon_{4}\right)= & A_{2} u^{2}+A_{4}^{P 1} u^{4}+\frac{1}{2} c_{s} \epsilon_{s}^{2}+\frac{1}{2} c_{44} \epsilon_{4}^{2} \\
& +\left(B_{s}^{P 1} \epsilon_{s}+B_{4}^{P 1} \epsilon_{4}\right) u^{2}+\mathcal{O}\left(u^{6}\right)
\end{aligned}
$$

where $u=u_{1}$ is the amplitude of the phonon displacement along $(1 \overline{1} 0), B_{s}^{P 1}=\frac{1}{4} B^{1}-2 B^{2}, B_{4}^{P 1}=B^{4}$, $\epsilon_{s}=\frac{1}{2}\left(\epsilon_{x x}+\epsilon_{y y}-2 \epsilon_{z z}\right)$, and $\epsilon_{4}=\epsilon_{x y}$. By eliminating $\epsilon_{1}$ and $\epsilon_{2}$ from Eq. (6) by the equilibrium condition $\partial \mathcal{F}^{P 1} / \partial \epsilon_{s}=\partial \mathcal{F}^{P 1} / \partial \epsilon_{4}=0$, one obtains

$$
\begin{gathered}
\epsilon_{s}=-\frac{B_{s}^{P 1} u^{2}}{c_{s}}, \epsilon_{4}=-\frac{B_{4}^{P 1} u^{2}}{c_{44}} \\
\widetilde{\mathcal{F}}^{P 1}(u)= \\
A_{2} u^{2} \\
+\left(A_{4}^{P 1}-\frac{B_{s}^{P 1^{2}}}{2 c_{s}}-\frac{B_{4}^{P 1^{2}}}{2 c_{44}}\right) u^{4}+\mathcal{O}\left(u^{6}\right) .
\end{gathered}
$$

Equation (8) shows that the coupling between the soft phonon and macroscopic strain renormalizes the fourthorder coefficient, making it large and negative, whenever $c_{s}$ or $c_{44}$ are small enough. Due to the ongoing softening of the shear constant, $c_{s}$, we find that at the softening pressure the fourth-order coefficient is negative, and we conclude that the transition must then be first order, occurring at a somewhat lower pressure (see the discussion at the end of Sec. II A). A similar behavior is observed along $P 2$. A thorough study of the transition might be performed by considering the expansion of the crystal energy up to sixth order in the order parameter, and by fitting the relevant coefficients to first-principles calculations, as it was done when the coupling to the strain was neglected. However the presence of many sixth-order invariants and the smallness of the computed energy differences make such a procedure impractical. We have preferred instead to concentrate on the $P 1$ line which is geometrically simpler and whose symmetry is compatible

TABLE VIII. Fourth-order polynomial invariants of the phonon-strain coupling.

\begin{tabular}{lc}
\hline \hline$Q_{4}^{1}(\mathbf{u}, \epsilon)$ & $\left(\epsilon_{x x}+\epsilon_{y y}+\epsilon_{z z}\right)\left(u_{1}^{2}+u_{2}^{2}+u_{3}^{2}+u_{4}^{2}+u_{5}^{2}+u_{6}^{2}\right)$ \\
$Q_{4}^{2}(\mathbf{u}, \epsilon)$ & $\left(2\left(u_{1}^{2}+u_{2}^{2}\right)-u_{3}^{2}-u_{4}^{2}-u_{5}^{2}-u_{6}^{2}\right) \times$ \\
& $\left(2 \epsilon_{z z}-\epsilon_{x x}-\epsilon_{y y}\right)+3\left(u_{3}^{2}+u_{4}^{2}-u_{5}^{2}-u_{6}^{2}\right)\left(\epsilon_{x x}-\epsilon_{y y}\right)$ \\
$Q_{4}^{3}(\mathbf{u}, \epsilon)$ & $\left(-2 u_{1} u_{2}-u_{3} u_{4}-u_{5} u_{6}\right)\left(\epsilon_{x x}-\epsilon_{y y}\right)+$ \\
& $\left(-u_{3} u_{4}+u_{5} u_{6}\right)\left(2 \epsilon_{z z}-\epsilon_{x x}-\epsilon_{y y}\right)$ \\
$Q_{4}^{4}(\mathbf{u}, \epsilon)$ & $\left(u_{1}^{2}-u_{2}^{2}\right) \epsilon_{x y}+\left(u_{3}^{2}-u_{4}^{2}\right) \epsilon_{y z}+\left(u_{5}^{2}-u_{6}^{2}\right) \epsilon_{x z}$ \\
\hline \hline
\end{tabular}

with the observed x-ray diffraction pattern of the highpressure phase. For this direction we have performed straight energy minimizations with respect to $u$ and $\epsilon$. By repeating such a minimization for different volumes, one directly obtains the equation of state of the crystal in the low-symmetry phase.

\section{Relative stability}

CsI and CsBr both display a softening of the $M_{5}^{-}$ phonon frequency and the ferroelastic instability responsible for the cubic-to-tetragonal transition, whereas $\mathrm{CsCl}$ does not show any tendency to phonon softening and a very weak tendency towards the ferroelastic instability. We conclude that the high-pressure stable phase of $\mathrm{CsCl}$ in the pressure range up to $\approx 100 \mathrm{GPa}$ is cubic $\mathrm{B2}$, whereas the relative stability of the tetragonal and orthorhombic phases must be checked for $\mathrm{CsBr}$.

We have performed complete energy minimizations with respect to atomic displacements and strain parameters for both the tetragonal and the newly determined orthorhombic structure. For CsI we have found a firstorder transition to the orthorhombic phase. All structural parameters, including volume, are discontinuous at the transition pressure $P_{\text {ort }}=21 \mathrm{GPa}$ (see Fig. 2 of Ref. 12). The volume discontinuity is however very weak $(\approx 0.1 \%) . b / a$ exhibits a weak dependence upon applied pressure, whereas $c / a$ has a more marked dependence. Such a different behavior can be explained by noting that $c / a-1$ is inversely proportional to the nearly vanishing shear constant $c_{s}$, whereas $b / a-1$ depends on $c_{44}$ which is regular in this pressure range. We find that cesium and iodine have slightly different displacements in the unit cell. As a consequence the space group of our final structure is $D_{2 h}^{5}$, different from the $C_{2 v}^{1}$ originally proposed by Mao et al. ${ }^{11}$ However, the diffraction pattern corresponding to the two groups is the same, and our proposed structure is compatible with the experimental data.

We have compared the enthalpies of the various phases and identified the transition pressures through the Maxwell construction. Results of this procedure are shown in Fig. 7 where the enthalpies of CsI and $\mathrm{CsBr}$ are shown for the tetragonal and orthorhombic phases relative to the cubic one. In agreement with experimental findings the high-pressure stable phase of CsI is the orthorhombic one (for pressures larger than $\approx 21 \mathrm{GPa}$ ). At variance with the case of $\mathrm{CsI}$, in $\mathrm{CsBr}$ the tetragonal structure turns out to be more stable all over the explored pressure range. The phonon softening is preceded by the cubic-to-tetragonal transformation at $P_{\text {tet }}=58 \mathrm{GPa}$, and we have verified that the tetragonal phase is always favored. So we can definitely assign the high-pressure phase of $\mathrm{CsBr}$ to be tetragonal. The cubic-to-tetragonal transition has been experimentally observed in $\mathrm{CsBr}$ at $53 \pm 2 \mathrm{GPa}$ corresponding to a residual volume $v_{\text {tet }} \approx 0.54 .^{4}$ As we have seen, $\mathrm{CsCl}$ does not manifest any phonon softening upon increasing pressure. This compound shows only a weak tendency to a tetragonal instability at $P_{\text {tet }} \approx 69 \mathrm{GPa}$, but the tetrag- 


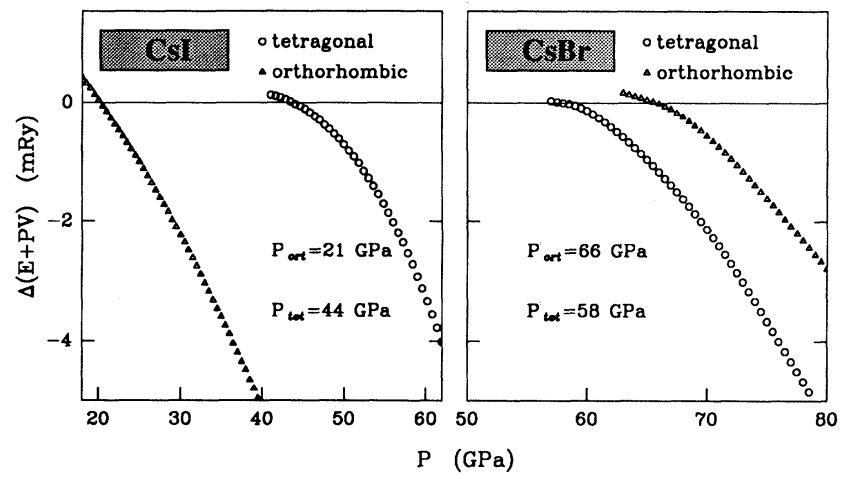

FIG. 7. Enthalpies of the orthorhombic and tetragonal phases, relative to the enthalpy of the cubic phase, as functions of the applied pressure in CsI and CsBr. $P_{\text {ort }}$ and $P_{\text {tet }}$ are the transition pressures from the cubic to the tetragonal and to the orthorhombic phases, respectively.

onal phase seems not to be stable at higher pressures. Our results are in agreement with this measurement. In $\mathrm{CsCl}$, a weak tetragonal instability has been observed experimentally at $65 \pm 5 \mathrm{GPa}$, but an extensive study at higher pressure is still lacking.

\section{CONCLUSIONS}

In this paper we have shown that a combined use of the Landau theory of phase transitions and accurate firstprinciples calculations based on DFT-LDA provide a reliable scheme for predicting the structural properties of ionic systems at high pressures. In the specific case of cesium halides, we have observed a trend in the highpressure phases which can be related with the polarizability of the anion. Our results indicate that the more polarizable is the anion the larger is the tendency towards a tetragonal instability of the cubic $B 2$ structure (passing from $\mathrm{CsCl}$ to $\mathrm{CsBr}$ ) and, for even larger polarizability towards an orthorhombic structure (from $\mathrm{CsBr}$ to CsI). Some investigations which we have performed also for other alkali halides indicate that when the cation is lighter (and hence less polarizable) than Cs, the stable high-pressure phase seems always to be the cubic $B 2$ one.

\section{ACKNOWLEDGMENTS}

We would like to thank S. de Gironcoli and P. Pavone for valuable discussions.
${ }^{1}$ E. Madelung, Phys. Z. 19, 524 (1918); P.O. Ewald, Ann. Phys. (Leipzig) 64, 253 (1921).

${ }^{2}$ H.K. Mao and P.M. Bell, Science 200, 1145 (1978).

${ }^{3}$ R. Reichlin, M. Ross, S. Martin, and K.A. Goettel, Phys. Rev. Lett. 56, 2858 (1986); Q. Williams and R. Jeanloz, ibid. 56, 163 (1986).

${ }^{4}$ T.-L. Huang and A.L. Ruoff, Phys. Rev. B 29, 1112 (1984); T.-L. Huang, K.E. Brister, and A.L. Ruoff, ibid. 30, 2968 (1984); Y.K. Vohra, K.E. Brister, S.T. Weir, S.J. Duclos, and A.L. Ruoff, Science 231, 1136 (1986).

${ }^{5}$ E. Knittle and R. Jeanloz, Science 223, 53 (1984); E. Knittle and R. Jeanloz, J. Phys. Chem. Solids 46, 1179 (1985); E. Knittle, A. Rudy, and R. Jeanloz, Phys. Rev. B 31, 588 (1985).

${ }^{6}$ P.W. Anderson and E.I. Blount, Phys. Rev. Lett. 14, 217 (1965).

${ }^{7}$ K. Asaumi, Phys. Rev. B 29, 1118 (1984).

${ }^{8}$ Y.K. Vohra, S.J. Duclos, and A.L. Ruoff, Phys. Rev. Lett. 54, 570 (1985).

${ }^{9}$ N.E. Christensen and S. Satpathy, Phys. Rev. Lett. 55, 600 (1985); S. Satpathy, N.E. Christensen, and O. Jepsen, Phys. Rev. B 32, 6793 (1985).

${ }^{10} \mathrm{~S}$. Baroni and P. Giannozzi, Phys. Rev. B 35, 765 (1987).

${ }^{11}$ H.K. Mao, Y. Wu, R.J. Hemley, L.C. Chen, J.F. Shu, and L.W. Finger, Science 246, 649 (1989); H.K. Mao, Y. Wu, R.J. Hemley, L.C. Chen, J.F. Shu, L.W. Finger, and D.E. Cox, Phys. Rev. Lett. 64, 1749 (1990).

12 M. Buongiorno Nardelli, S. Baroni, and P. Giannozzi, Phys. Rev. Lett. 69, 1069 (1992).

${ }^{13}$ L.D. Landau and E.M. Lifshitz, Statistical Physics (Pergamon, Oxford, 1980), Part I, Chap. XIV.

${ }^{14}$ See, for instance, W.E. Pickett, Comput. Phys. Rep. 9, 115 (1989).
${ }^{15}$ H. Hellmann, Einführung in die Quantenchemie (Deuticke, Leipzig, 1937); R.P. Feynman, Phys. Rev. 56, 340 (1939).

${ }^{16}$ O.H. Nielsen and R.M. Martin, Phys. Rev. B 32, 3780 (1985).

${ }^{17}$ S. Baroni, P. Giannozzi, and A. Testa, Phys. Rev. Lett. 58, 1861 (1987).

${ }^{18}$ S. Baroni, P. Giannozzi, and A. Testa, Phys. Rev. Lett. 59, 2662 (1987).

${ }^{19}$ U. von Barth and R. Car (unpublished). This scheme consists essentially of a fitting minimization of the squared differences between the atomic all-electron and pseudoeigenvalues and eigenfunctions (beyond a given core radius $r_{c}$ ), as functions of a few parameters on which the pseudopotential is made to depend.

${ }^{20}$ S.G. Louie, S. Froyen, and M.L. Cohen, Phys. Rev. B 26, 1738 (1982).

${ }^{21}$ D.M. Ceperley and B.J. Alder, Phys. Rev. Lett. 45, 566 (1980).

22 J. Perdew and A. Zunger, Phys. Rev. B 23, 5048 (1981).

${ }^{23}$ H.J. Monkhorst and J.P. Pack, Phys. Rev. B 13, 5188 (1976).

${ }^{24}$ F.D. Murnaghan, Proc. Natl. Acad. Sci. U.S.A. 30, 244 (1944).

25 S. Baroni and R. Resta, Phys. Rev. B 33, 7017 (1986); S. de Gironcoli, S. Baroni, and R. Resta, Phys. Rev. Lett. 62, 2843 (1989).

${ }^{26}$ K. Reinitz, Phys. Rev. 123, 1615 (1961).

${ }^{27}$ R.W.G. Wyckoff, Crystal Structures (R.E. Krieger Publishing Company, Malabar, 1982), Chap. III.

${ }^{28}$ R.K. Singh, H.N. Gupta, and M.K. Agrawal, Phys. Rev. B 17, 894 (1978).

${ }^{29}$ R.P. Lowndes and D.H. Martin, Proc. R. Soc. London, Ser. A 308, 473 (1969). 
${ }^{30}$ W.Bührer and W. Hälg, Phys. Status Solidi 46, 679 (1971).

${ }^{31}$ R.P. Lowndes, Phys. Rev. B 1, 2754 (1970).

${ }^{32}$ S. Rolandson and G. Raunio, Phys. Rev. B 4, 4617 (1971).

${ }^{33}$ A.A.Z. Ahmad, H.G. Smith, N. Wakabayashi, and M.K.
Wilkinson, Phys. Rev. B 6, 3956 (1972).

${ }^{34}$ J.S. Kim, D.M. Hatch, and H.T. Stokes, Phys. Rev. B 33, 1774 (1986). 